\title{
Labeless And Reversible Immunosensor Assay Based Upon An Electrochemical Current-Transient Protocol
}

\author{
Sarah Grant ${ }^{\#}$, Frank Davis*, Jeanette A. Pritchard ${ }^{+}$, Karen A. Law ${ }^{*}$, Seamus P. J. Higson ${ }^{*, 1}$, \\ and Timothy D. Gibson ${ }^{\#+}$
}

*Institute of Biosciences and Technology, Cranfield University, Silsoe, Beds, MK45 4DT, UK.

\# School of Biochemistry and Molecular Biology, University of Leeds, Irene Manton Building, Leeds, LS2 9JT, UK.

${ }^{\dagger}$ T \& D Technology Ltd., Stanley, Wakefield, West Yorkshire, WF3 4AA, UK.

${ }^{+}$Applied Enzyme Technology Ltd., Monmouth House, Mamhilad Park, Pontypool, NP4 0HZ.

${ }^{1}$.Corresponding author. Fax (+44) 01525 863433, email s.p.j.higson@cranfield.ac.uk 


\section{ABSTRACT}

A novel labeless and reversible immunoassay based upon an electrochemical current-transient protocol is reported which offers many advantages in comparison to classical immunobiochemical analyses in terms of simplicity, speed of response, reusability and possibility of multiple determinations.

Conducting polypyrrole films containing antibodies against 1) Bovine Serum Albumin (BSA) and 2) Digoxin were deposited on the surface of platinum electrodes to produce conductive affinity matrices having clearly defined binding characteristics. The deposition process has been investigated using ${ }^{125}$ I labelled anti-digoxin to determine optimal fabrication protocols. Antibody integrity and activity, together with non-specific binding of antigen on the conducting matrix have also been investigated using tritiated digoxin to probe polypyrrole/anti-digoxin films. Amperometric responses to digoxin were recorded in flow conditions using these films, but the technique was limited in use mainly due to baseline instability.

Anti-BSA - polypyrrole matrices were investigated in more detail in both flow and quiescent conditions. No observable response was found in flow conditions, however under quiescent conditions (in non-stirred batch cell), anti-BSA - polypyrrole films have been demonstrated to function as novel quantitative chronoamperometric immuno-biosensors when interrogated using a pulsed potential waveform. The behaviour of the electrodes showed that the antibody/antigen binding and/or interaction process underlying the response observed was reversible in nature, indicating that the electrodes could be used for multiple sensing protocols.

Calibration profiles for BSA demonstrated linearity for a concentration range of 0-50 ppm but tended towards a plateau at higher concentrations. Factors relating to replicate sensor production, sample measurement and reproducibility are discussed. 


\section{INTRODUCTION}

Since the principle of immunoassay was first published by Yallow and Berson in 1959 [1] there has been an exponential growth in both the range of analytes to which the technique has been successfully applied and the number of novel assay designs. Developments in immunoassay techniques have led to improvements in user-friendliness and sensitivity compared with many other traditional techniques such as mass spectrometry and gas liquid chromatography. The development of enzyme-labelled immunoanalytical techniques e.g. Enzyme-Linked Immuno Sorbent Assay (ELISA) has also provided analytical tests without the safety risks associated with radiolabelling-based techniques.

A direct immunosensor that does not rely on the use of a detectable label could lead to simpler assay formats and ideally lower times of detection. The rapid measurement of analytes of clinical significance e.g. towards various disease markers could in turn permit earlier intervention, which in a medical setting is frequently of utmost importance. A reusable and rapid detection system would, moreover, allow for continuous real-time measurement, so helping to maintain optimal homeostatic conditions. The development of reversible immunosensors towards measurement of the antibody-antigen reaction in real time has been limited by the nature of the antibody-antigen reactions, many of which are not readily reversible. Antibodies bind solely to their antigens and achieve specificity via a complex series of multiple non-covalent bonds. In such processes there are no newly formed molecules, protons or electrons that are easily detectable, which has limited the development of direct antibody affinity-type sensors. Additionally, since the antibody-antigen binding event is often irreversible (affinity binding constants typically range from $10^{5}$ to $10^{11} \mathrm{~mol}$. $\mathrm{l}^{-1}$ ) many contemporary immunosensors are only of use for “one-shot” analyses and therefore must be disposable in nature.

The incorporation of antibodies into conducting polymer films was first reported by John et al [2]. Anti-human serum albumin (anti-HSA) was incorporated into a polypyrrole film, which 
was galvanostatically polymerised onto a platinum wire substrate. When grown in the absence of a counterion, a poor polymeric film, both in appearance and electrochemical properties, resulted suggesting that the presence of a counterion was necessary for the polymerisation process to be successful. Amino acid analysis of the polymer using a leucine marker showed that approximately $0.1 \% \mathrm{w} / \mathrm{v}(0.2 \mu \mathrm{g})$ of the antibody was incorporated into the matrix. When the pyrrole anti-HSA electrode was exposed to $50 \mu \mathrm{g} \mathrm{ml}^{-1} \mathrm{HSA}$ for ten minutes, a new reduction peak was observed at a potential of approximately $+600 \mathrm{mV}$ vs. Ag/AgCl. This peak increased in magnitude after a further thirty minutes in the same solution and it was suggested this could be due to an antibody/antigen interaction with the polymer. Further work by the same group gave rise to reports of a reversible real-time immunosensor [2]. Using a square wave potential pulse waveform, a transient amperometric response was measured that appeared proportional to the concentration of antigen in the sample, with detection limits being reported as low as 5 ppm [3]. This technique of pulsed amperometric detection was transferred to other analytes, including p-cresol [4], Thaumatin [5] and polychlorinated biphenyls [6].

Other workers have attempted to reproduce the work on HSA reported in these papers with little success [7], however during our investigations into the production of conducting polymer antibody loaded films an antibody response was observed to a pulsed waveform and it is from here that the results described in this communication were developed. Also it must be noted that potentiometric deposition protocols almost always resulted in electroactive films, whereas galvanostatic protocols tended to give overoxidised films that were not electroactive, even though the observed coverage of the sensor surface appeared to be more complete. This may be a function of the antibodies used, however, in most reports to date, pure antibodies have not been prepared (most commercial sources have other proteins added as stabilisers) and other components such as nitrate ions have been included in the polymerisation matrix to enable 
electroconductive, galvanostatically prepared films to be produced. In this work only potentiostatically deposited films were used in the electrochemical interrogation experiments described. It has been reported by Yuan et al that overoxidised polypyrrole films containing $\mathrm{K}_{4}\left[\mathrm{Fe}\left(\mathrm{CN}_{6}\right)\right]$ and formate dehydrogenase $+\mathrm{NAD}$ were produced at potentials of $800 \mathrm{mV}$ over a 20 minute period [8], thus the potential of $900 \mathrm{mV}$ used in these studies would clearly produce non-conducting overoxidised films when deposition was carried out galvanostatically over the timescales used.

Antibody-antigen interactions are by their very nature complex and the reproducible response characteristics of immunosensors requires that the affinity reaction occurring is minimally perturbed by the fabrication procedure. It has been shown in our work here that antibodies may be successfully incorporated into conducting polymer films by entrapment in a growing polypyrrole film with no detrimental effect to antibody activity. The process of immunosensor manufacture is an important issue that is often overlooked and we have taken time to focus on the methods of immunosensor production, together with the use of ${ }^{125} \mathrm{I}$ as a radiolabel to directly quantify the amount of antibody entrapped in the conducting films produced. The whole sensor was counted, together with the appropriate controls to give a clear result of antibody incorporation. Similarly we have direct evidence that the binding of antigen to the corresponding antibody occurs by using a tritium labelled antigen and incubating this with antibodies entrapped in conducting polypyrrole films. This part of the work was carried out using the purer antibody preparation, anti-digoxin IgG to minimise interferences from extraneous contaminants.

Our initial interrogation studies were based on the pulsed amperometry technique described for the detection of human serum albumin [3] using both an anti-BSA and the antidigoxin immunosensors produced in the first part of the work and a Dionex PAD II detector. 
This technique was originally used for the measurement of carbohydrates via their amperometric oxidation at the surface of either a gold or platinum electrode $[9,10]$.

Sadik and Wallace adapted this pulsed amperometry methodology for the monitoring of antibody-antigen interactions on a polymer-modified electrode [3]. One of the main limitations to the development of an immunosensor is the large binding energy between the antibody and antigen, which in many instances give rise to interactions that are not readily reversible. The findings by Sadik and Wallace suggested, however, that by pulsing the potential between two suitably chosen values, the surface of the working electrode could be regenerated and in this way a sensor might be used for further measurements. A Dionex PAD-II system was used for the polarisation of antibody-loaded polypyrrole films via a pulsed potential waveform, in conjunction with a time-dependent current monitoring regime. It was reported that this technique produced a direct immunosensor that could be used for multiple analyses with typical response times of less than one minute $[3,11,12]$.

While this work showed great promise we have found great difficulty in reproducing the results reliably using both the anti-BSA and anti-digoxin immunosensors produced and encountered problems of day-to-day reproducibility of manufacture of individual sensors, as well as baseline instability of the sensors produced.

To attempt to understand the source of these irreproducibilities, we also interrogated immunosensors produced using anti-BSA IgG using a repeating polarising waveform in batch mode and collected the entire current transient using an oscilloscope. The results obtained have definitively proved that a reproducible signal is contained within the current transient obtained and in this paper we have gone some way towards developing the reliability of the technique as an immunosensors format. 


\section{MATERIALS AND METHODS}

\section{Chemicals and Reagents}

All reagents were analytical grade unless otherwise stated. Concentrated sulphuric acid was purchased from Fisher Scientific UK (Leicestershire, UK). Bovine serum albumin (BSA), pyrrole, sodium dichromate, platinum gauze, platinum foil, bovine anti-IgG and sheep anti-BSA antibodies were supplied by Sigma-Aldrich Company Ltd. (Dorset, England). Anti-digoxin antibodies were purified using an affinity column technique according to the method described by Grant [13], to give an essentially pure preparation of lyophilised sheep anti-digoxin IgG. Starting material was immunised sheep serum kindly donated by Therapeutic Antibodies Ltd. ${ }^{125}$ I labelled anti-digoxin IgG was prepared by Dr P. A. Milner, School of Biochemistry and Molecular Biology, University of Leeds, using the purified antibodies prepared previously. Tritium labelled digoxin was obtained from New England Nuclear. Screen-printed electrodes were purchased from Gwent Electronics Materials Ltd. Deionised water was obtained from a Millipore Milli-Q purification system.

\section{Apparatus}

Sonication of cleansed platinum wire electrodes was performed using a Kerry Sonicator. A Thompson E Series Ministat potentiostat was used for deposition of antibody-polypyrrole films onto platinum wire.

A wall-jet configuration flow cell was kindly donated by Professor Lo Gorton (Department of Analytical Chemistry, University of Lund), which consisted of a platinum wire counter electrode, a Ag/AgCl reference electrode and a platinum working electrode constructed “in house”. A Gilson Minipulse 3 peristaltic pump, a Gallenkamp Datatrace chart recorder and an Anachem Ltd Universal Valve Switching Module Injection system were interfaced to the system. 
Polymerisation of pyrrole unlabelled immunosensors, was performed using a Sycopel AEW2 computer-controlled potentiostat that recorded all output parameters directly via software version 1996-97. The deposition protocol was carried out in a 3-electrode cell incorporating reference, working and auxiliary electrodes (Fig.1). Labelled immunosensors were prepared in a controlled environment using the same deposition protocols, but an EG\&G Model 362 manual potentiostat was used for electropolymerisation.

A Dionex Pulsed Amperometric Detector-II (PAD-II) was linked to a Gallenkamp Datatrace Chart Recorder. The Dionex PAD-II system operates as an integral potential squarewave generator, potentiostat and current data recorder. Thus potential, E1 and E2 can be changed and in this way the square waveform defined, although the current was always recorded during the last 20ms of E2 (Fig.3).

Platinum wires were inserted into a purpose-built plastic case (Fig.1). A silicon seal was created around the platinum and a flush edge achieved using epoxy resin surrounding the circumference of the protruding platinum wire. A multi-core wire was soldered to the external end of the platinum wire to create a secure connection. Injection of samples into the flow-cell system was accomplished using a Universal Valve Switching Module purchased by Anachem Ltd.

\section{Preparation of Platinum Electrodes}

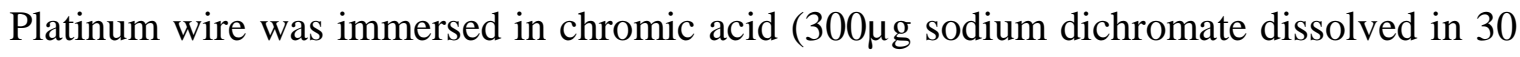
ml concentrated sulphuric acid) for 10 minutes followed by a thorough rinsing in de-ionised (ELGA-Milli Q) water and sonication for 5 minutes in de-ionised water.

Planar platinum electrodes were used as supplied from Gwent Electronic Materials Ltd. and used directly from the packaging material to avoid contamination. 


\section{Pyrrole Purification.}

Pyrrole was distilled twice under vacuum before use using a Quick Fit micro-distillation apparatus in batches of approximately $10 \mathrm{ml}$. After distillation the pyrrole was placed in a darkened container and deoxygenated by bubbling nitrogen through the solution. Pyrrole was stored in a sealed container at $-20{ }^{\circ} \mathrm{C}$ and was warmed to room temperature before use.

\section{Preparation of Polypyrrole/Anti-BSA Solution}

Anti-BSA solution was dialysed for 2 hours against ELGA water to remove low molecular weight contaminants and then $200 \mu \mathrm{l}$ (equivalent to $480 \mu \mathrm{g}$ protein) was added to $5 \mathrm{ml}$ of $0.5 \mathrm{M}$ pyrrole solution in ELGA water $(173 \mu \mathrm{l}$ of distilled pyrrole into $5 \mathrm{ml}$ water) and stirred gently to fully disperse the reagents.

\section{Preparation of Polypyrrole/Anti-digoxin Solution}

Anti-digoxin / pyrrole solution used to prepare the platinum wire electrodes was prepared from 20mg anti-digoxin lyophilisate (10.4 mg protein the remainder $\mathrm{NaCl}$ ) added to 5

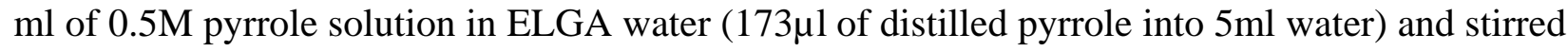
gently to fully dissolve the reagents.

Solutions used to prepare planar platinum electrodes were prepared using the same procedure but at a lower concentration of $100 \mu \mathrm{g}$ anti-digoxin per ml. Radio-labelled experiments

using anti-digoxin used this concentration and $1 \mu \mathrm{g}$ of ${ }^{125} \mathrm{I}$ labelled anti-digoxin added to $3 \mathrm{ml}$ of pyrrole / unlabelled anti-digoxin solution (300 $\mu \mathrm{g}$ in total). In these experiments pyrrole concentration was varied between $0.1 \mathrm{M}$ and $0.5 \mathrm{M}$ in $0.1 \mathrm{M}$ steps. 
Deposition of Antibody-Containing Polypyrrole Films onto Platinum Electrodes

Polypyrrole films containing anti-BSA or anti-digoxin were deposited onto cleaned, platinum wire electrodes previously described or planar platinum electrodes of $3.14 \mathrm{~mm}^{2}$ working area using a potential of $900 \mathrm{mV}$ vs. Ag/AgCl with a platinum gauze auxiliary electrode. To avoid contamination of the $\mathrm{Ag} / \mathrm{AgCl}$ reference electrode and any leakage of the reference electrode electrolyte into the polymerisation formulations, a double junction reference electrode was used in all polymerisation protocols, Fig.1. A deposition time of 10 minutes was employed as standard, however for the time course experiments 5, 10 and 15 minutes were evaluated. After growth the electrode was washed in ELGA water three times to ensure a clean surface. Potentiostatic deposition protocols were used to ensure the polypyrrole films produced remained electroactive as it was previously known [8] and confirmed by us that galvanostatically produced films were often overoxidised and therefore non-electroactive when the antibodies described were used. Galvanostatically deposited films were prepared for comparative purposes using a current density of $1 \mathrm{~mA} \mathrm{~cm}{ }^{-2}$.

Determination of calibration curve for ${ }^{125}$ I labelled anti-digoxin polypyrrole films.

Pyrrole $(134 \mu \mathrm{l})$ was mixed with $0.1 \mathrm{M}$ potassium chloride $(100 \mu \mathrm{l})$ and made up to $4 \mathrm{ml}$ with Milli Q grade water and films were deposited at a constant potential of $950 \mathrm{mV} \mathrm{Vs} \mathrm{Ag} / \mathrm{AgCl}$ (Sat $\mathrm{KCl}$ ) for 5 minutes. In total 18 films were grown by this method using 3 batches of the solution just described. Various amounts of the ${ }^{125}$ I anti-digoxin were pipetted onto the surface of the prepared pyrrole films and allowed to dry. The screen-printed electrodes were then counted for 60 seconds in a Alloc 1470 Wizard Automatic gamma counter in the pre-described orientation and an appropriate calibration curve was constructed, Fig.3. Due to the relatively short half-life of 56 days for the ${ }^{125}$ I isotope, this calibration curve was retained and recounted with every set of experiments performed. 


\section{Measurement of Digoxin}

Digoxin was dissolved in ELGA water with exhaustive stirring to give $100 \mu \mathrm{g}^{-L^{-1}}$ stock solutions. Standard solutions were prepared to the desired concentrations from these stocks. Platinum working electrodes were placed into a 3-electrode, purpose built cell, through which analytes and/or buffer could be pumped by means of a peristaltic pump. Diluent (ELGA water) was passed through the cell at a flow rate of $1 \mathrm{ml} \mathrm{min}^{-1}$ for thirty minutes prior to each set of sample injections to allow a stable baseline to be established. The Dionex PAD-II apparatus was connected and $20 \mu \mathrm{L}$ samples were injected into the flow, still at $1 \mathrm{ml} \mathrm{min}^{-1}$. The Dionex apparatus was programmed to repetitively generate a pulsed potential starting at $0.0 \mathrm{~V} v s$. $\mathrm{Ag} / \mathrm{AgCl}$ (for $60 \mathrm{~ms}$ duration) before stepping to $+0.7 \mathrm{~V}$ for $540 \mathrm{~ms}$ and then returning to $0.0 \mathrm{~V}$ to begin the next cycle. This long pulse duration is a function of the Dionex design which is for carbohydrate analysis $[9,10]$. The current was then sampled repetitively during the last 20ms of $\mathrm{E}_{2}(+0.7 \mathrm{~V})$. Output from the Dionex apparatus was monitored using a Gallenkamp Datatrace chart recorder.

\section{Data Capture for Total Current Transients}

For interrogation of the whole waveform from the anti-BSA immunosensors, a Thurlby and Thander TG215 Function generator was used in conjunction with a Thompson E Series Ministat Potentiostat and the output monitored using Picoscope capture software. Comparable parameters to those created using the Dionex PAD-II apparatus were achieved, producing a waveform between 0 and $0.7 \mathrm{~V}$ vs $\mathrm{Ag} / \mathrm{AgCl}$ at a frequency of $10 \mathrm{~Hz}$ (equivalent to a pulse width of $50 \mathrm{~ms}$ ) across a 3-electrode unstirred, batch type cell to remove any interferences from solution flow. We are not trying to replicate the polarisation/interrogation regime of the Dionex but rather are trying to understand and record the nature of a current transient following pulse polarisation 
of the system. These measurements cannot be directly compared to earlier FIA work [3] due to the different sample introduction regime, but because this experiment gives rise to current transients related to analyte (antigen) concentration in a repeatable manner, the implication is still the same that the antibody-antigen reaction appears to be reversible in nature.

\section{Analytical Protocol}

The platinum working electrode, $\mathrm{Ag} / \mathrm{AgCl}$ reference electrode and platinum gauze counter electrode were placed into a small glass vessel and covered with purified ELGA water (5 $\mathrm{ml}$ ). The working electrode was repeatedly polarised by means of the staircase waveform for three minutes to establish a baseline. The water was then replaced with $5 \mathrm{ml}$ of a known concentration of BSA sample and the pulsed waveform monitored for 3 minutes. The cell was washed three times with water before re-establishing a baseline for the next determination. BSA concentrations ranged from 0 to 100ppm with multiple current transients being recorded per concentration and for varying time intervals.

\section{RESULTS AND DISCUSSION}

Antibody Loading of Polypyrrole Films

Direct incorporation of anti-digoxin antibodies into growing polypyrrole films has been

demonstrated using ${ }^{125} \mathrm{I}$ labelled anti-digoxin added to the polymerisation solution before deposition. Increasing the concentration of pyrrole in the polymerisation solution has a small effect on the amount of anti-digoxin incorporated into the film, with higher concentrations giving more consistent and even films on visual observation, which is an indicator of better film-to-film reproducibility. Increasing the polymerisation time from 5 minutes up to 15 minutes also influenced the overall amount of anti-digoxin incorporated. 
The results are summarised in Fig.4. Based on these results and the fact that a higher pyrrole concentration gave more even coverage of the planar platinum electrodes used, a concentration of 0.5M pyrrole, with a polymerisation time of 10 minutes was used throughout the remainder of the work. The same charge was consumed during galvanostatic and potentiostatic growth as confirmed by charge integration techniques for films containing both antibody and the chloride controls.

During polymerisation the anti-digoxin is entrapped into the growing film to give an affinity surface as evidenced by the selective affinity reaction between the anti-digoxin entrapped and the radiolabelled digoxin shown in Fig.5. The incorporation of a non-selective bovine antiIgG as a control gave virtually no counts in each of the pyrrole concentrations tested, clearly showing non-specific binding of the labelled digoxin was minimal. The anti-digoxin results shown in Fig.5 are averages of triplicate films produced at each concentration of pyrrole. It is clear that the anti-digoxin incorporated into the growing polypyrrole films is immuno-active and available for antigen binding events to occur at the surface of the deposited films. Scanning electron microscopy of prepared films indicated that the planar platinum electrodes displayed surface roughness yet deposition by potentiostatic protocols covered the platinum surface with a thin layer of polypyrrole incorporating entrapped antibody. Galvanostatic protocols gave films that appeared thicker, but as described previously often these were non-electroactive and therefore useless as immunosensors. Films prepared using chloride as a counter ion were much thicker in nature and had a different morphology. Electron micrographs of the deposited films are shown in Fig.6.

\section{Antigen Detection Using DC Pulsed Amperometry}

Attempts have been made by other workers to repeat the work of Sadik and Wallace [3], however great difficulty has been experienced in attaining reproducible results [7]. There have, 
however, been several reports in the literature of the technique being successfully exploited by the same authors e.g. for the detection of human serum albumin [3], p-cresol [4], Thaumatin [5] and polychlorinated biphenyls [6]. The authors have informed us that the majority of these results were obtained using one particular Dionex PAD-II machine (personal communication Prof G G Wallace 1999). It appears that attempts to transfer the technique across to a nominally similar machine of identical type and model have been only partially successful and in most other cases, where the same type of electrochemical interrogation techniques have been used, other academic groups have not been able to reproduce the results reported. Direct discussions with Dionex (Technical Support Dept, 1999) revealed that differing machines that "appear to be identical" do in fact monitor current at different relative timings within the pulsing cycle, even if they are set at what should be identical sampling according to the control dials on each individual machine. We later determined this, as will be described in forthcoming discussions, to be one source of the discrepancies reported by different groups [7].

Fig.7a shows representative chronoamperometric current transients in response to injection of a $1 \mathrm{mg} . \mathrm{l}^{-1}$ digoxin sample into the flow cell containing an anti-digoxin antibody immunosensor, giving rise to peak currents of 20nA. Addition of differing concentrations of digoxin to the same type of electrode produced on a different day but with exactly the same deposition parameters, Fig.7b gave one series of peaks that indicated a concentration dependant response. The baseline drift can be clearly seen and the signal response to the same concentration of digoxin is different between the different electrodes addressed. These two series of responses were the only ones clearly observed using the Dionex PAD II system, therefore it was concluded that the Dionex interrogation of antibody loaded sensors was insufficiently reliable for immunosensors interrogation.

In both cases, injections of distilled water gave no signal, which tended to indicate that the signal was due to the antibody response to the digoxin injected. These responses were only 
observed on a sporadic basis however, as 1) the baseline tended to drift erratically and could not be compensated for and 2) the responses were irregular and non-reproducible as can be seen for the differential responses for $1 \mathrm{mg} . \mathrm{l}^{-1}$ digoxin in Figs $7 \mathrm{a}$ and $7 \mathrm{~b}$. No clear results were observed from the anti-BSA immunosensors using this form of immunosensor interrogation. Clearly the method was unsuitable as an analytical technique, however on a positive note there was some sort of signal generation occurring.

\section{Detection of BSA Using Current Transient Analysis}

With the understanding of the Dionex sampling regimen, and with the current transients occurring at slightly different relative timings depending on the machine being used, experiments were conducted to pulse the immunosensor surface and then to record the whole current transient response. Due to easier availability of anti-BSA the development of a usable analytical protocol was aimed towards anti-BSa immunosensors.

As described within the experimental section, the antibody-containing films were potentiostatically pulsed at a frequency of $10 \mathrm{~Hz}$ from 0 to $0.7 \mathrm{~V}$ vs $\mathrm{Ag} / \mathrm{AgCl}$ and the current output for the entire waveform recorded using a Thompson E Series Ministat Potentiostat in conjunction with Picoscope capture software. At the beginning of each polarisation, not the end of the pulse, the observed current curve was shown to rapidly rise to a maximum which decayed (Fig. 8a). The greatest consistency in the output from successive peak to peak was found to be at the initiation of the pulse, with the output just before the pulse was terminated being rather irreproducible. Using this initial current response, a BSA calibration curve was constructed by the measurement of the initial peak currents from the current transients traces, Fig.8b. The calibration profile showed near-linear behaviour between 0 and 50ppm BSA $\left(r^{2}=0.996\right)$, at which point the curve started to tend towards a plateau. The original baseline was always re-established upon washing of the electrode with deionised water between samples. The calibration curve 
constructed was from a single anti-BSA electrode that was washed successively between BSA additions and which remained connected to the signal generator at all times during the experiment, i.e. the surface was continually pulsed.

The technique of constantly pulsing films has been used by other workers. They found that resultant signals were more easily interrogated at shorter time-scales following pulsing in agreement with our findings [14,15].

The results obtained were very clear in comparison to the Dionex interrogation of the immunosensors and in control experiments performed using anti-BSA loaded films; distilled water additions did not produce any change in the current observed, and therefore the evidence is that the current transients observed are due to an interaction of the anti-BSA in the immunosensors with the BSA solution added [11,12]. Also it must be noted that the differing concentrations of BSA used were not introduced to the immunosensor surface sequentially, but on a random basis to ensure the responses recorded did not result from an attenuation of the sensor surface. Samples were measured on ten electrodes with multiple injections (minimum 4 per sample) over several days. In all cases the response profiles were found to be reproducible, displaying a maximum standard deviation of $5 \mathrm{pA}\left(5 \times 10^{-3} \mathrm{nA}\right)$.

The reversibility of the sensor in generating the reproducible responses to each of the BSA concentrations introduced has clearly been demonstrated in this case, (minimum 4 repeats for each sensor). Ten sensors were used and the spread of data is shown by the error bars in Fig.8b, and correspond to a maximum standard deviation of 0.5. This is consistent with the earlier reports where reversibility was observed and multiple assays could be carried out on a single immunosensors.

Control experiments in which BSA samples were exposed to antibody-free polypyrrole films, made with chloride doped polypyrrole showed no increase or change in current output, confirming that the currents observed were due in some way to the antibody-antigen interaction 
$[11,12]$. In each case the reported results corresponded to peak currents, observed immediately following the beginning of a polarisation pulse for the first five pulses. This approach of capturing the entire current transient proved significant in furthering our understanding towards attaining reliable and reproducible interrogation into these effects.

This approach contrasted significantly with the method of the Dionex PAD-II system within our laboratory, which sampled the current 520ms after the initial polarisation of the surface. Since the current transients tend rapidly towards a plateau at this point, monitoring a faradaic reaction generated by the antibody and antigen would prove to be difficult, indeed it can be shown that the interaction giving rise to this effect occurs over a time scale of a few tens to hundreds of milliseconds. To further complicate matters we subsequently determined from Dionex that apparently similar machines manufactured at different times actually monitor current at slightly different relative times during the timespan of a pulse and we believe in the main, that this accounts why different groups have previously obtained widely differing results from apparently identical instrumentation. In essence each machine is internally calibrated to be accurate but different machines are not absolutely electronically identical with each other, therefore the settings that are set on one machine may be subtly different from another set on the supposed same settings, leading to the incorrect interrogation of identical immunosensor surfaces. It should be realised in this context that the Dionex instrument was produced for the measurement of steady state current for the determination of carbohydrates and other oxidisable analytes and for this purpose such relative timing differences are irrelevant - even though they prove critical to work involving antibody loaded polypyrrole films.

The actual source of the signal is as yet unclear, but it has been observed that the polypyrrole matrix has to be electroconductive for a signal to be observed. Non-conductive, overoxidised matrices give no measurable responses on addition of antigen. This would indicate that the actual signal generation is linked to the matrix, rather than a direct product of the 
antibody - antigen interaction alone and further work into the mechanism of signal generation will be reported in a future publication.

In summary, BSA has been shown to bind reversibly and measurably to antibody modified electrodes and gave excellent calibration profiles over a working concentration range of 0-50 mg. $\mathrm{l}^{-1}$, so demonstrating the principle of antibody - and indeed practical significance behind this approach for a novel electrochemical immunosensor format. 


\section{CONCLUSIONS}

We have definitively proved incorporation of antibody into growing polypyrrole films and that such antibodies retain affinity properties for their antigens using radiolabelled probes. Non-specific adsorption of the radiolabelled probes has been found to be extremely low. Potentiometrically deposited films were more often electroactive than galvanostatically deposited films, and retention of the electroactivity of the films was necessary to observe any signal generation.

We have determined that subtle differences in the commercial Dionex machines are most likely responsible for the variability of results reported in the literature for different antibody polypyrrole electrode systems. A methodology based upon the captivity and interrogation of current transients upon square waveform potential excitation has allowed for a novel generic approach for a reversible immuno-electrochemical sensor regime. Demonstration of this approach has been initially shown by the fabrication of an anti-Bovine Serine Albumin - polypyrrole modified platinum electrode based sensor with a practical working concentration range of 0-100 mg. $\mathrm{l}^{-1}$ BSA, and which showed a linearity of $\mathrm{r}^{2}=0.966$ over a $0-50$ mg. $\mathrm{l}^{-1}$ concentration range. Reversibility of the antibody - antigen interaction has be demonstrated using a single immunosensor continuously pulsed at a frequency of $10 \mathrm{~Hz}$, between $0.0 \mathrm{~V}$ and $+0.7 \mathrm{~V}$ vs $\mathrm{Ag} / \mathrm{AgCl}$. 


\section{ACKNOWLEDGEMENTS}

We wish to thank the EPSRC for a studentship for S. Grant and the British Textiles Technology Group for sponsorship of this CASE award and also the Biotechnology and Biological Sciences Research Council, BBSRC, (U.K.) for part funding of this work as part of the Centre for BIOARRAY Innovation. 


\section{REFERENCES}

1. R. S. Yallow and S. A. Berson. Nature. 184 (1959) 1648-1649.

2. R. John., M. Spencer, G. G. Wallace, M. R. Smyth, Anal. Chim. Acta. 249 (1991) 381-385.

3. O.A. Sadik, G. G. Wallace, Anal. Chim. Acta. 279 (1993) 209-212.

4. D. Barnett, G. G. Wallace, D. G. Laing, S. Skopec, O. A. Sadik. Anal. Lett.. 27 (1994) 24172429.

5. O. A. Sadik, M. J. John, G. G. Wallace, D. Barnett, C. Clarke, D. G. Laing, Analyst. 119 (1994) 1997-2000.

6. S. Bender, O. A. Sadik, Environmental Science Technology. 32 (1998) 788-797.

7. J. N.Barisci, D. Hughes, A. Minett, G. G. Wallace, Anal. Chim. Acta. 371 (1998) 39-48.

8. Y J Yuan, H Zhao, G G Wallace and S B Adeloju. Electrochemistry Communications 2 (2000) 27-31.

9. P. T. Kissinger, Anal. Chem. 49 (1977) A447-452.

10. R. J. Rucki, Talanta. 27 (1980) 147-152.

11. A. Sargent and O. A. Sadik, Anal. Chim. Acta. 376 (1998) 125-131.

12. A. Sargent and O. A. Sadik, Electrochimica. Acta. 44 (1999) 4667-4675.

13. S. Grant, PhD Thesis (2000), University of Leeds.

14. A. Sargent T. Loi, S. Gal and O. A. Sadik, J. Electroanalytical. Chem. 470 (1999) 144-156.

15. O. A. Sadik, H. Xu, E. Gheorgiu, D. Andreescu, C. Balut, M. Gheorgiu and D. Bratu, Anal. Chem. 74 (2002) 3142-3150. 
Figure 1

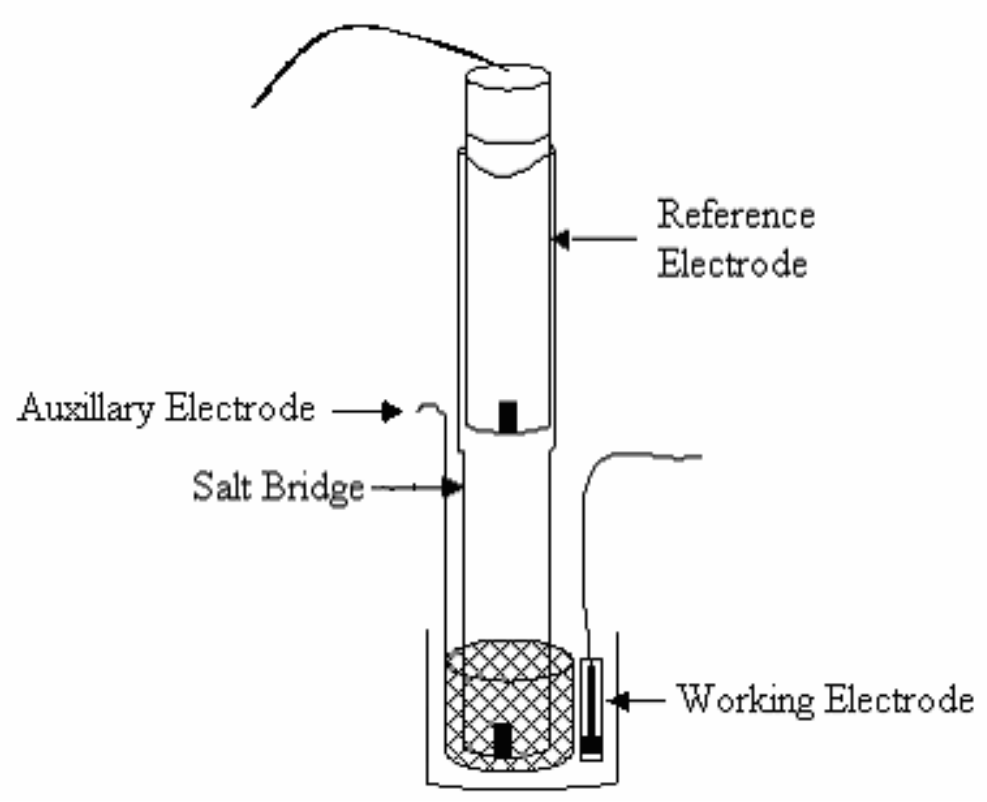


Figure 2

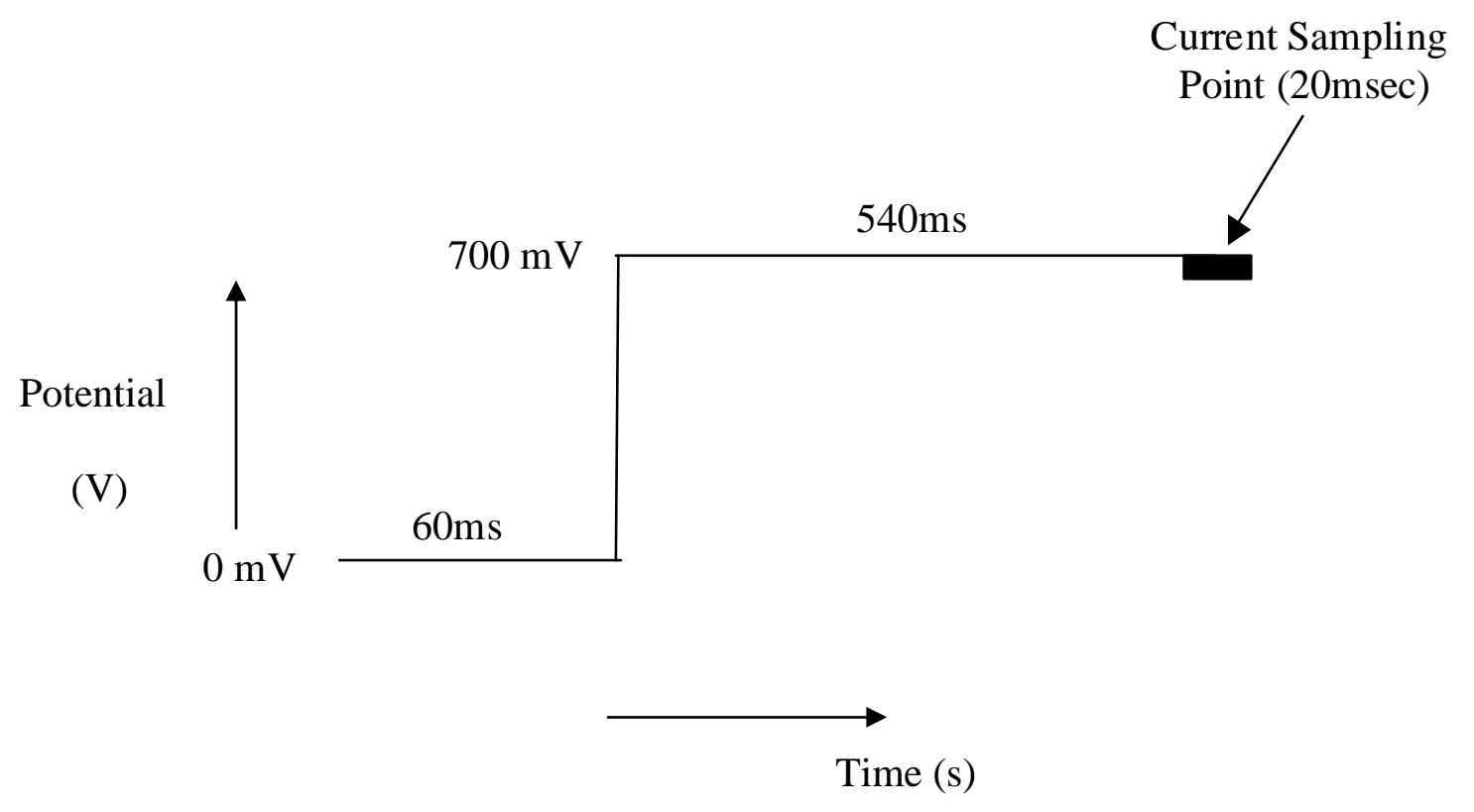


Figure 3

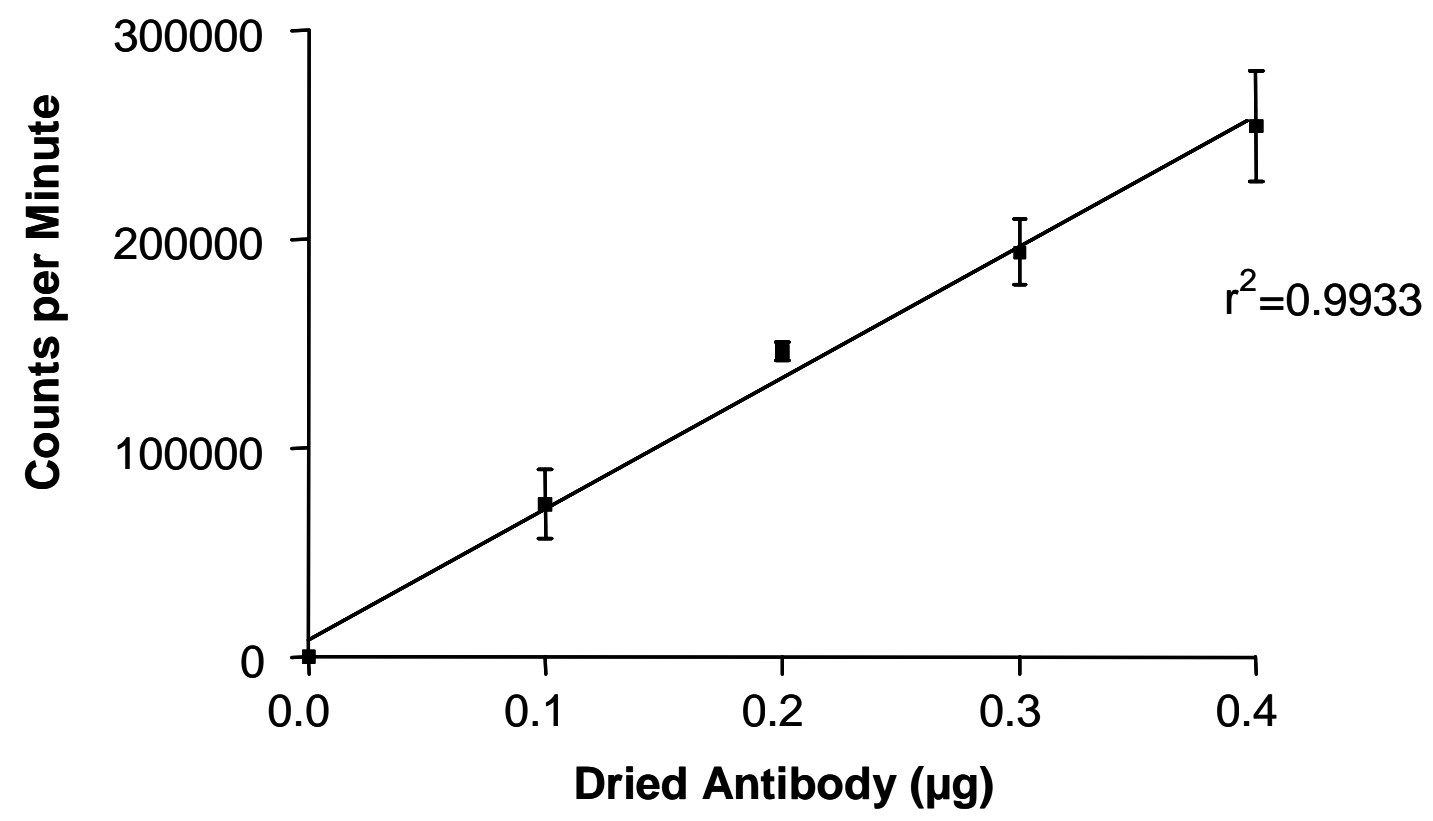


Figure 4

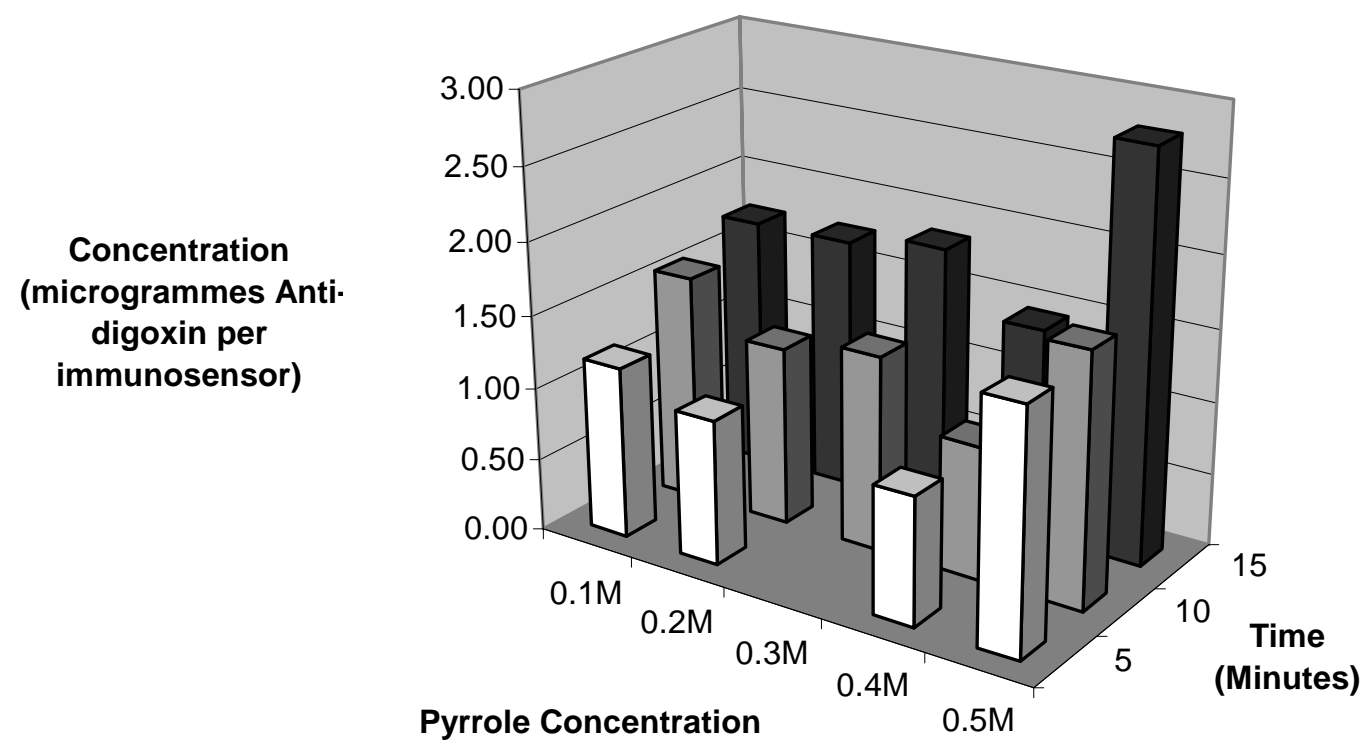


Figure 5

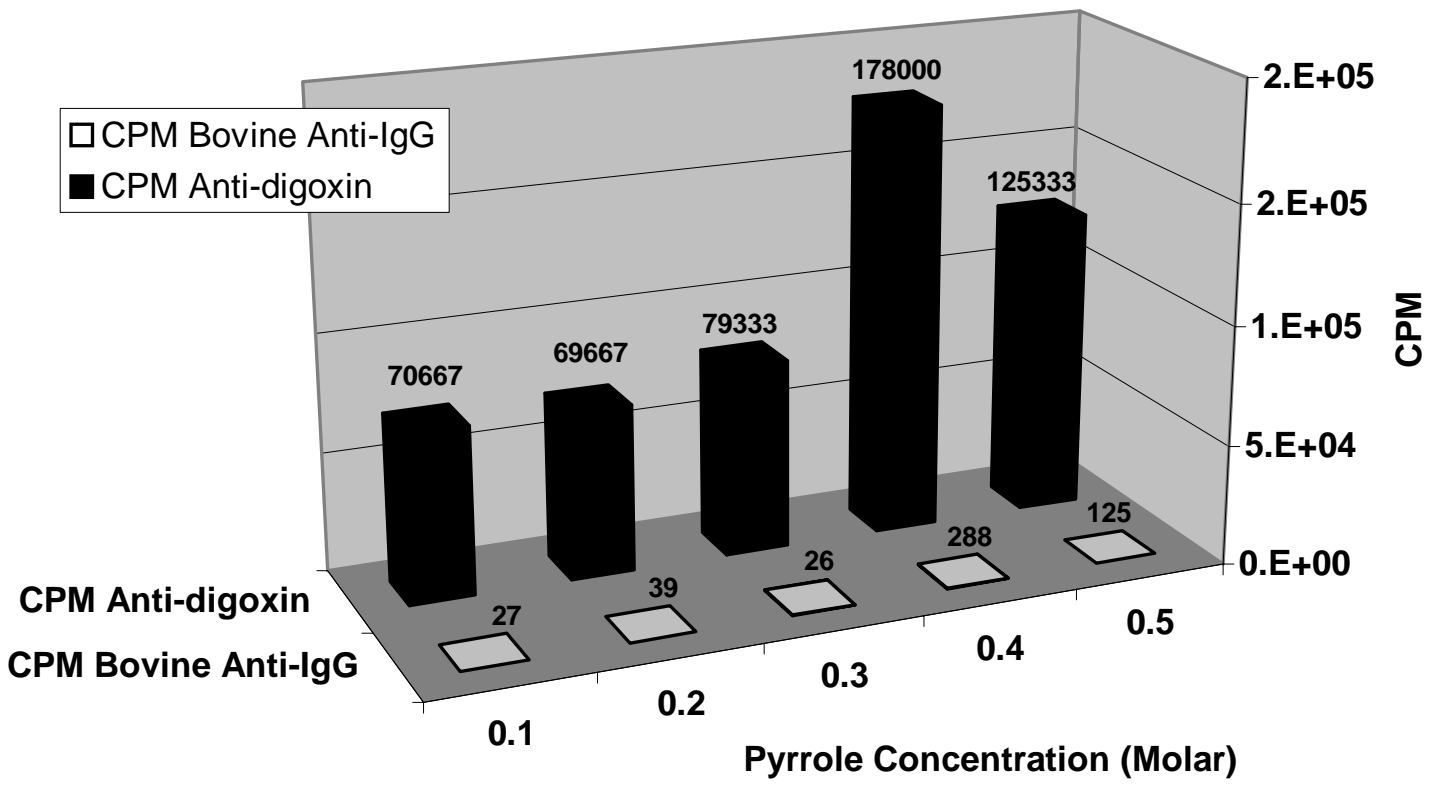


Figure 6

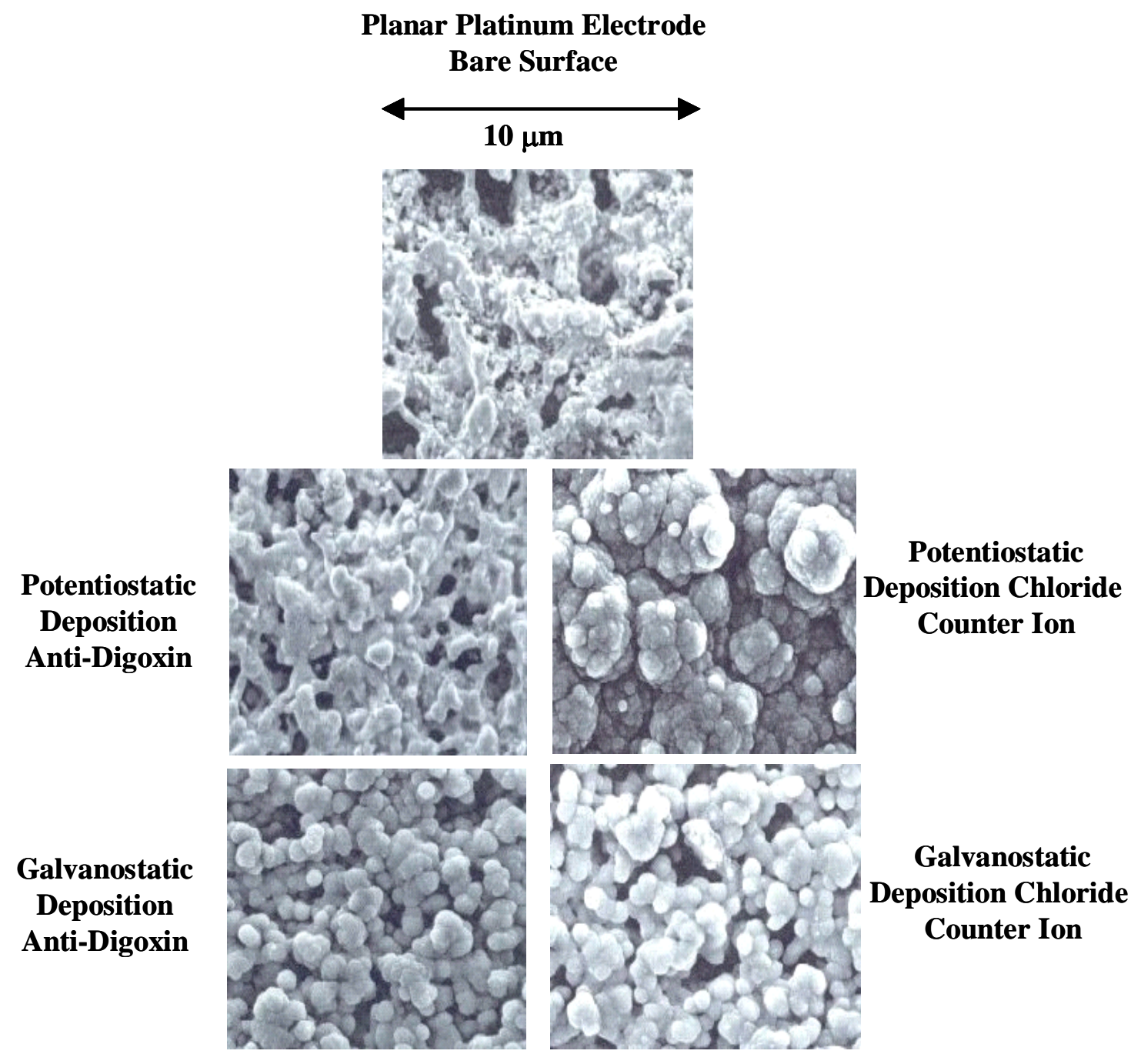


Figure 7a

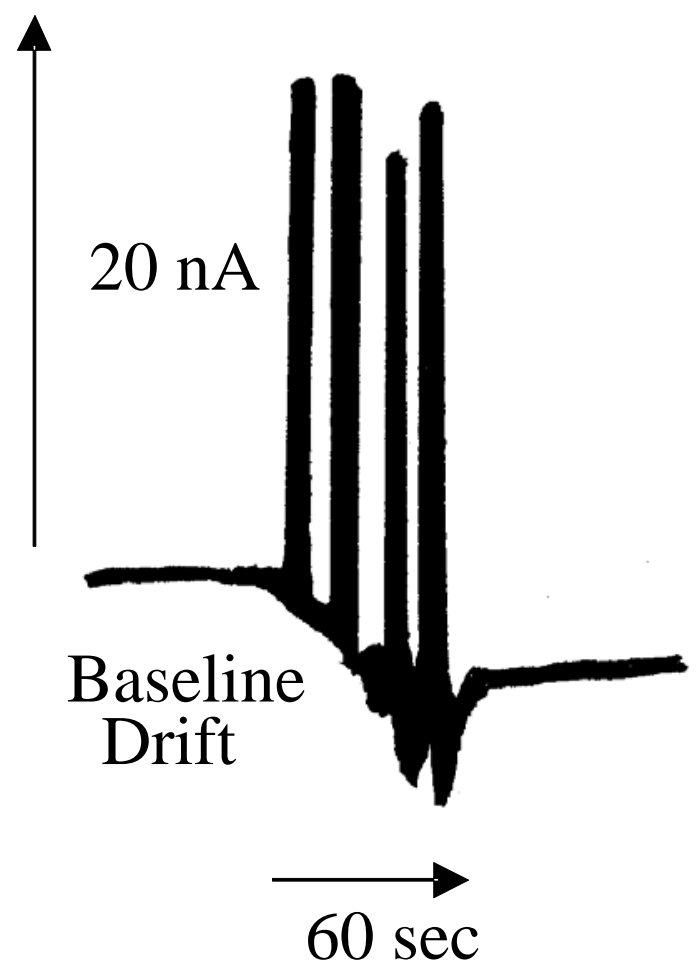


Figure 7b

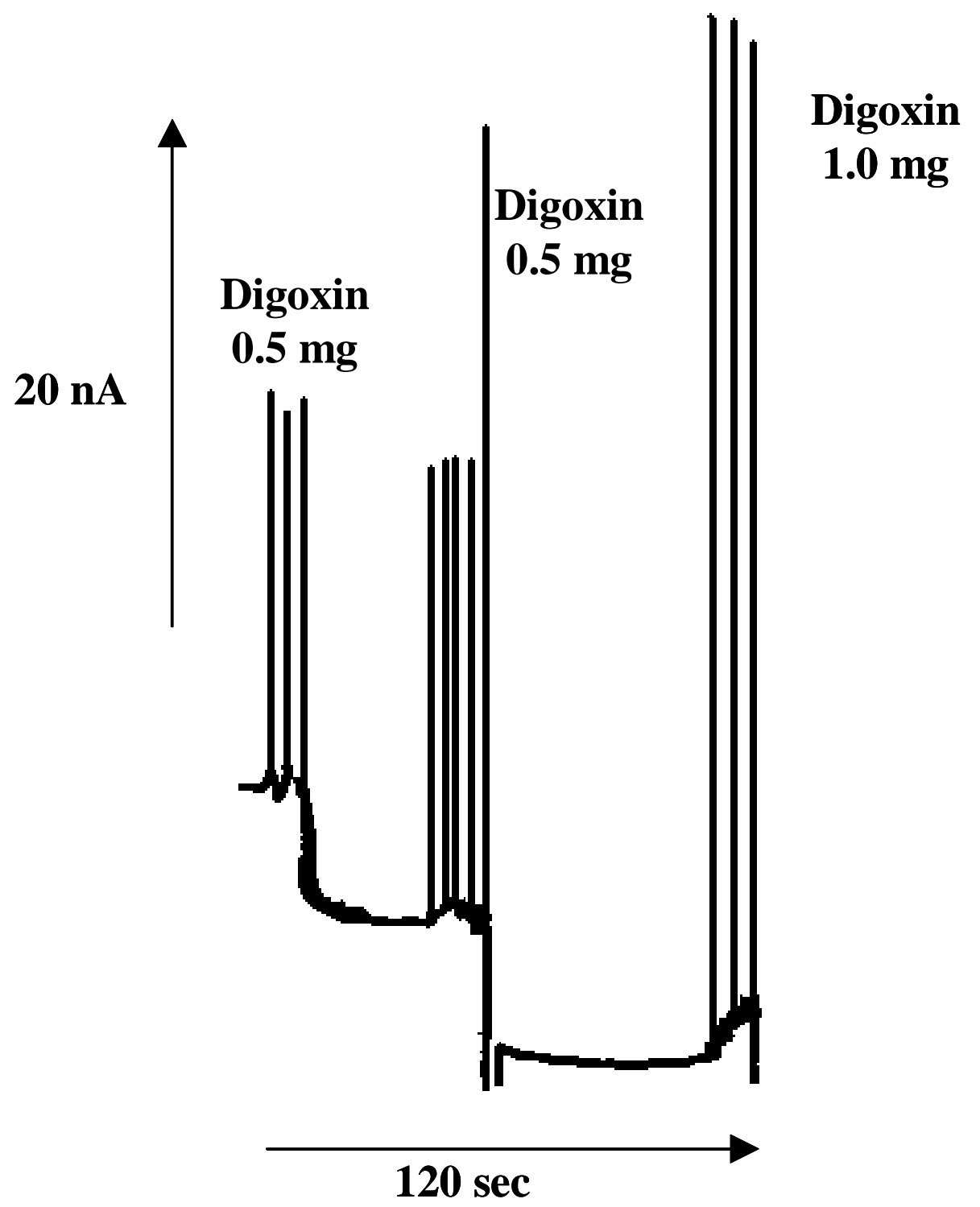


Figure 8
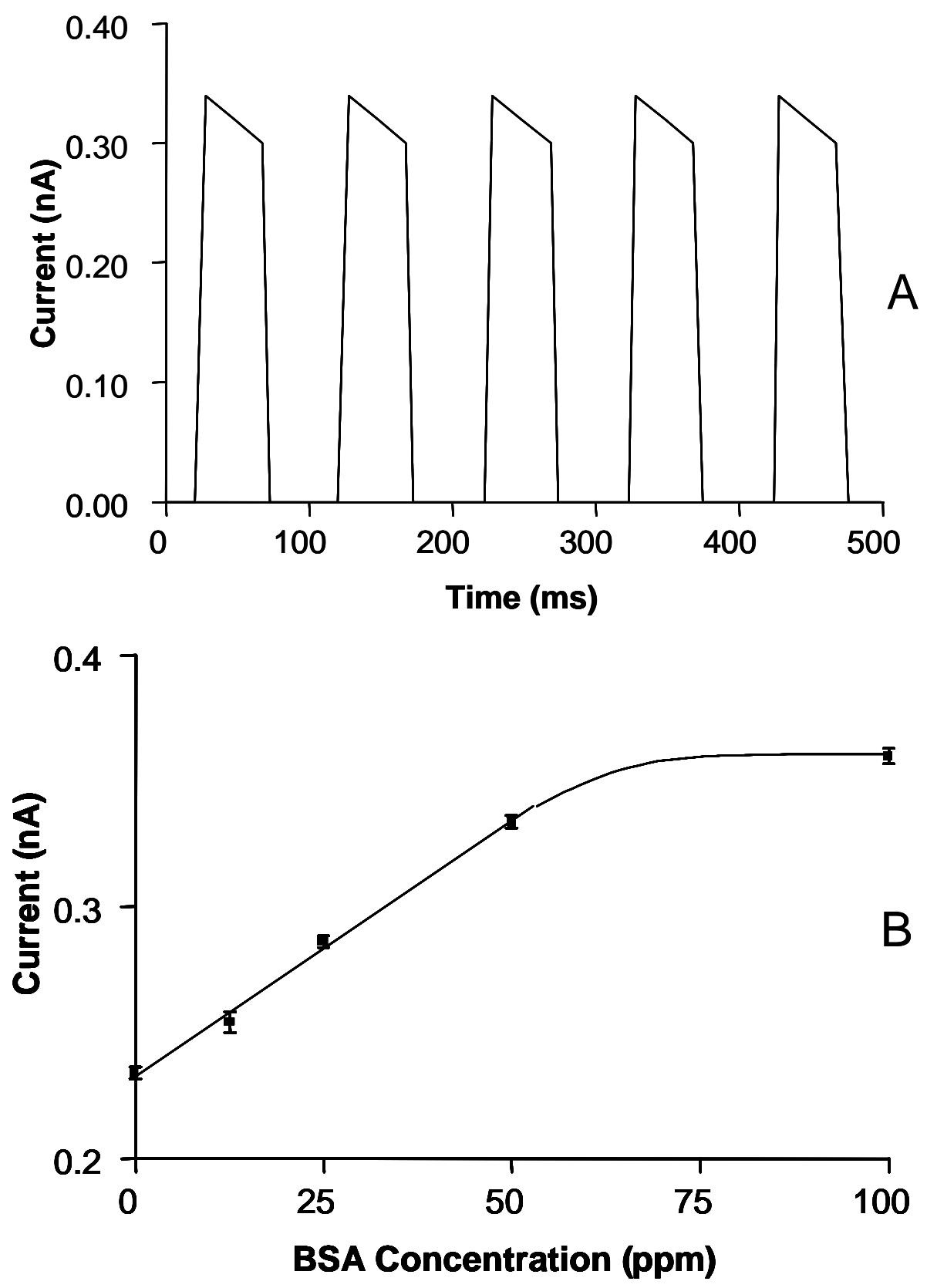


\section{Legends for figures.}

Fig.1. Schematic diagram of the 3-electrode cell used in the polymerisation protocols.

Fig.2. Pulsed potential waveform used by the Dionex PAD II system for the detection of digoxin in flow systems.

Fig.3. Calibration curve for ${ }^{125}$ I labelled anti-digoxin electrodes to enable estimation of antibody loading for different deposition protocols.

Fig.4. Potentiometric deposition of anti-digoxin - polypyrrole films, effect of polymerisation time and initial pyrrole concentration.

Fig.5. Adsorption of tritiated digoxin onto antibody loaded polypyrrole films.

Fig.6. SEM images of electrodeposited films.

Fig.7a and 7b Current transients for two different anti-digoxin loaded polypyrrole films produced on different days upon exposure to aqueous digoxin injections in flow conditions and periodic square wave polarisation as shown in Fig.2.

Fig.8 (a). Typical current transient (Picoscope) trace in the presence of BSA. (b). Peak current responses for an anti-BSA loaded polypyrrole film for varying concentrations of BSA. 